\title{
Generalized Central Factorial Numbers with Odd Arguments
}

\author{
Youmna H. Zaid, F. A. Shiha, B. S. El-Desouky* \\ Department of Mathematics, Faculty of Science, Mansoura University, Mansoura, Egypt \\ Email: youmnazed@yahoo.com,fshiha@mans.edu.eg, ‘b_desouky@yahoo.com
}

How to cite this paper: Zaid, Y.H., Shiha, F.A. and El-Desouky, B.S. (2020) Generalized Central Factorial Numbers with Odd Arguments. Open Journal of Modelling and Simulation, 8, 61-72.

https://doi.org/10.4236/ojmsi.2020.83005

Received: March 21, 2020

Accepted: June 14, 2020

Published: June 17, 2020

Copyright (อ 2020 by author(s) and Scientific Research Publishing Inc. This work is licensed under the Creative Commons Attribution International License (CC BY 4.0).

http://creativecommons.org/licenses/by/4.0/

\begin{abstract}
In this paper, we consider $r$-generalization of the central factorial numbers with odd arguments of the first and second kind. Mainly, we obtain various identities and properties related to these numbers. Matrix representation and the relation between these numbers and Pascal matrix are given. Furthermore, the distributions of the signless $r$-central factorial numbers are derived. In addition, connections between these numbers and the Legendre-Stirling numbers are given.
\end{abstract}

\section{Keywords}

Generalized Central Factorial Numbers with Odd Arguments, Pascal Matrix, Legendre-Stirling Numbers

\section{(c) (i) Open Access}

\section{Introduction}

Riordan ([1], pp. 213-217), defined the central factorial numbers of the first and second kind $t(n ; k)$ and $T(n ; k)$, respectively

$$
\begin{aligned}
& x \prod_{i=1}^{n-1}\left(x+\frac{n}{2}-i\right)=\sum_{k=0}^{n} t(n, k) x^{k}, \\
& x^{n}=\sum_{k=0}^{n} T(n, k) x \prod_{i=1}^{k-1}\left(x+\frac{k}{2}-i\right) .
\end{aligned}
$$

Equivalently, the $t(n, k)$ and $T(n, k)$ are determined by the recurrence relations

$$
\begin{aligned}
& t(n, k)=t(n-2, k-2)-\left(\frac{n-2}{2}\right)^{2} t(n-2, k), \quad 2 \leq k \leq n, \\
& T(n, k)=T(n-2, k-2)+\left(\frac{k}{2}\right)^{2} T(n-2, k), \quad 2 \leq k \leq n,
\end{aligned}
$$


with $t(n, n)=T(n, n)=1$ and $t(n, k)=T(n, k)=0$ for $n<k$. If $n$ and $k$ are both odd, then $t(n, k)$ and $T(n, k)$ are not integers. For more details on the central factorial numbers, see Butzer et al. [2].

Kim et al. [3] extended $T(n, k)$ to the $r$-central factorial numbers of the second kind, $r$ is a non-negative integer

$$
\frac{1}{k !} \mathrm{e}^{r t}\left(\mathrm{e}^{\frac{t}{2}}-\mathrm{e}^{\frac{-t}{2}}\right)^{k}=\sum_{n=k}^{\infty} T_{r}(n+r, k+r) \frac{t^{n}}{n !} .
$$

In [4], the central factorial numbers with even arguments of both kinds are given by

$$
u(n, k)=t(2 n, 2 k) \text { and } \quad U(n, k)=T(2 n, 2 k),
$$

and the central factorial numbers with odd arguments of both kinds are given by

$$
v(n, k)=4^{n-k} t(2 n+1,2 k+1) \text { and } V(n, k)=4^{n-k} T(2 n+1,2 k+1) .
$$

Note that $v(n, k)$ and $V(n, k)$ are integers for all $n, k \geq 0$. The combinatorial interpretations of these numbers can be found in [4] and the references therein.

Recently, Shiha [5] introduced the $r$-cental factorial numbers with even arguments of the first (resp. the second) kind $u_{r}(n, k)$ (resp. $U_{r}(n, k)$ ), and introduced many properties and identities for these numbers. For all integers $n, k \geq 0$,

$$
\begin{aligned}
& \prod_{\ell=0}^{n-1}\left(x-\ell^{2}\right)=\sum_{k=0}^{n} u_{r}(n, k)(x+r)^{k}, \\
& (x+r)^{n}=\sum_{k=0}^{n} U_{r}(n, k) \prod_{\ell=0}^{k-1}\left(x-\ell^{2}\right) .
\end{aligned}
$$

In the next, we consider a polynomial generalization of the cental factorial numbers with odd arguments of the first and second kind, which we will denote by $v_{r}(n, k)$ and $V_{r}(n, k)$, respectively. The distribution of the signless $r$-central factorial numbers with odd arguments of the first kind is derived. Moreover, we give many properties of these new numbers, including a new and interesting connection between these numbers and the Legendre-Stirling numbers.

\section{The Generalized Central Factorial Numbers with Odd Arguments}

Definition 1. Given integers $r, n \geq 0$, the arrays $v_{r}(n, k)$ and $V_{r}(n, k)$ are defined by

$$
\prod_{\ell=0}^{n-1}\left(x-(2 \ell+1)^{2}\right)=\sum_{k=0}^{n} v_{r}(n, k)(x+r)^{k},
$$

and

$$
(x+r)^{n}=\sum_{k=0}^{n} V_{r}(n, k) \prod_{\ell=0}^{k-1}\left(x-(2 \ell+1)^{2}\right) .
$$


In particular, if $r=0$, the numbers $v_{r}(n, k)$ are reduced to $v(n, k)$ and $V_{r}(n, k)$ are reduced to $V(n, k)$.

These numbers satisfy the following orthogonality relation:

$$
\sum_{k=\ell}^{n} v_{r}(n, k) V_{r}(k, \ell)=\sum_{k=\ell}^{n} V_{r}(n, k) v_{r}(k, \ell)=\delta_{n \ell} .
$$

The numbers $v_{r}(n, k)$ and $V_{r}(n, k)$ satisfy the following two-term recurrence relations.

Theorem 1. The arrays $v_{r}(n, k)$ and $V_{r}(n, k)$ for $n \geq k \geq 0$ are satisfy the recurrence

$$
v_{r}(n+1, k)=v_{r}(n, k-1)-\left((2 n+1)^{2}+r\right) v_{r}(n, k), \quad n, k \geq 1,
$$

and

$$
V_{r}(n+1, k)=V_{r}(n, k-1)+\left((2 k+1)^{2}+r\right) V_{r}(n, k), \quad n, k \geq 1,
$$

with $v_{r}(n, 0)=(-1)^{n} \prod_{\ell=0}^{n-1}(2 \ell+1)^{2}+r, \quad V_{r}(n, 0)=(1+r)^{n}$ and $v_{r}(0, k)=V_{r}(0, k)=\delta_{k, 0}$ for $n, k \geq 0$.

Proof. From (7), we have

$$
\begin{aligned}
& \sum_{k=0}^{n+1} v_{r}(n, k)(x+r)^{k}=\prod_{\ell=0}^{n}\left(x-(2 \ell+1)^{2}\right) \\
& =\prod_{\ell=0}^{n-1}\left(x-(2 \ell+1)^{2}\right)\left(x-(2 n+1)^{2}\right) \\
& =\prod_{\ell=0}^{n-1}\left(x-(2 \ell+1)^{2}\right)\left(x+r-r-(2 n+1)^{2}\right) \\
& =\prod_{\ell=0}^{n-1}\left(x-(2 \ell+1)^{2}\right)(x+r)-\left(r+(2 n+1)^{2}\right) \prod_{\ell=0}^{n-1}\left(x-(2 \ell+1)^{2}\right) \\
& =\sum_{k=0}^{n} v_{r}(n, k)(x+r)^{k+1}-\left(r+(2 n+1)^{2}\right) \sum_{k=0}^{n} v_{r}(n, k)(x+r)^{k} \\
& =\sum_{k=1}^{n+1} v_{r}(n, k-1)(x+r)^{k}-\left(r+(2 n+1)^{2}\right) \sum_{k=0}^{n} v_{r}(n, k)(x+r)^{k}
\end{aligned}
$$

Equating the coefficients of $(x+r)^{k}$ on both sides, we obtain Equation (10). For $k=0$, we find

$$
v_{r}(n+1,0)=-\left((2 n+1)^{2}+r\right) v_{r}(n, 0), \quad n=0,1, \cdots
$$

Successive application gives $v_{r}(n, 0)=(-1)^{n} \prod_{\ell=0}^{n-1}(2 \ell+1)^{2}+r$. The proof for (11) is similarly.

Moreover, we derive explicit formulas and further recurrences satisfied by $v_{r}(n, k)$ and $V_{r}(n, k)$ by using the following theorem.

Proposition 2. (Mansour et al. [6]) Suppose that the array $\{y(n, k)\}_{n, k \geq 0}$ is defined by

$$
y(n, k)=y(n-1, k-1)+\left(a_{n-1}+b_{k}\right) y(n-1, k), \quad n, k \geq 1
$$

with $y(n, 0)=\prod_{\ell=0}^{n-1}\left(a_{\ell}+b_{0}\right)$ and $y(0, k)=\delta_{0 k}$, for all $n, k \geq 0$, where $\left\{a_{j}\right\}_{j \geq 0}$ and $\left\{b_{j}\right\}_{j \geq 0}$ are given sequences with the $b_{j}$ distinct, then 


$$
y(n, k)=\sum_{j=0}^{k}\left(\frac{\prod_{\ell=0}^{n-1}\left(b_{j}+a_{\ell}\right)}{\prod_{\ell=0, \ell \neq j}^{k}\left(b_{j}-b_{\ell}\right)}\right), \forall n, k \in \mathbb{N},
$$

and

$$
y(n, k)=\sum_{j=k}^{n} y(j-1, k-1) \prod_{\ell=j}^{n-1}\left(a_{\ell}+b_{k}\right) .
$$

Theorem 3. For any integer $0 \leq k \leq n$,

$$
\begin{gathered}
V_{r}(n, k)=\frac{1}{2^{2 k}(2 k+1) !} \sum_{j=0}^{k}(-1)^{k+j}\left(\begin{array}{c}
2 k+1 \\
k-j
\end{array}\right)\left((2 j+1)^{2}+r\right)^{n}(2 j+1) . \\
v_{r}(n, k)=\sum_{\ell=k}^{n}(-1)^{n-\ell} v_{r}(\ell-1, k-1) \prod_{i=\ell}^{n-1}\left((2 i+1)^{2}+r\right) . \\
V_{r}(n, k)=\sum_{\ell=k}^{n} V_{r}(\ell-1, k-1)\left((2 k+1)^{2}+r\right)^{n-\ell} .
\end{gathered}
$$

Proof. Setting $a_{j}=0$ and $b_{j}=(2 j+1)^{2}+r$ for all $j$ in (13), then

$$
V_{r}(n, k)=\sum_{j=0}^{k} \frac{\left((2 j+1)^{2}+r\right)^{n}}{\prod_{i=0, i \neq j}^{k}\left((2 j+1)^{2}-(2 i+1)^{2}\right)} .
$$

Since $\prod_{i=0, i \neq j}^{k}\left((2 j+1)^{2}-(2 i+1)^{2}\right)=(-1)^{k+j} 2^{2 k} \frac{(k+j+1) !(k-j) !}{2 j+1}$, then

$$
\begin{aligned}
V_{r}(n, k) & =\sum_{j=0}^{k}(-1)^{k+j} \frac{\left((2 j+1)^{2}+r\right)^{n}}{2^{2 k}(k+j+1) !(k-j) !}(2 j+1) \\
& =\frac{1}{2^{2 k}(2 k+1) !} \sum_{j=0}^{k}(-1)^{k+j} \frac{(2 k+1) !\left((2 j+1)^{2}+r\right)^{n}}{2^{2 k}(k+j+1) !(k-j) !}(2 j+1) \\
& =\frac{1}{2^{2 k}(2 k+1) !} \sum_{j=0}^{k}(-1)^{k+j}\left(\begin{array}{c}
2 k+1 \\
k-j
\end{array}\right)\left((2 j+1)^{2}+r\right)^{n}(2 j+1) .
\end{aligned}
$$

For (16), set $a_{i}=-(2 i+1)^{2}+r, b_{k}=0$ in (14), and for (17), set $a_{i}=0$, $b_{k}=(2 k+1)^{2}+r$ in (14).

To get the exponential generating function of $V_{r}(n, k)$, multiply both sides of (15) by $\frac{t^{n}}{n !}$ and summing over $n \geq k$,

$$
\sum_{n=k}^{\infty} V_{r}(n, k) \frac{t^{n}}{n !}=\frac{1}{2^{2 k}(2 k+1) !} \sum_{j=0}^{k}(-1)^{k+j}\left(\begin{array}{c}
2 k+1 \\
k-j
\end{array}\right)(2 j+1) \mathrm{e}^{\left((2 j+1)^{2}+r\right) t} .
$$

\section{The Distribution of $\left|v_{r}(n, k)\right|$}

The signless $r$-central factorial numbers of odd arguments of the first kind is defined as

$$
\mathfrak{v}_{r}(n, k)=(-1)^{n-k} v_{r}(n, k)=\left|v_{r}(n, k)\right| .
$$

Theorem 4. The array $\mathfrak{v}_{r}(n, k)$ has a Poisson-binomial distribution. 
Proof. Define the random variables $X_{n}, n=1,2, \cdots$, such that

$$
P\left(X_{n}=k\right)=\frac{\mathfrak{v}_{r}(n, k)}{\sum_{k=0}^{n} \mathfrak{v}_{r}(n, k)}=\frac{\mathfrak{v}_{r}(n, k)}{\prod_{\ell=0}^{n-1}\left(1+r+(2 \ell+1)^{2}\right)}, \quad k=0,1, \cdots, n .
$$

The probability generating function of $X_{n}$ is given by

$$
\begin{aligned}
E\left(s^{X_{n}}\right) & =\sum_{k=0}^{n} s^{k} P\left(X_{n}=k\right)=\prod_{\ell=0}^{n-1} \frac{s+r+(2 \ell+1)^{2}}{1+r+(2 \ell+1)^{2}} . \\
& =\prod_{\ell=0}^{n-1}\left(1-\frac{1}{1+r+(2 \ell+1)^{2}}+\frac{s}{1+r+(2 \ell+1)^{2}}\right) .
\end{aligned}
$$

Then $X_{n}$ can be represented as a total number of successes in $n$ independent Bernoulli trials where

$$
p_{i}=\frac{1}{1+r+(2 i+1)^{2}}
$$

is the probability of success at trial $i$. Thus, the random variable $X_{n}$ has a Poisson-binomial distribution and hence, the array $\mathfrak{v}_{r}(n, k)$.

\section{Generating Function Formulas}

In this section, we give the generating function formulas and some related identities for the numbers $v_{r}(n, k)$ and $V_{r}(n, k)$.

Theorem 5. If $n \geq 0$, then

$$
\begin{aligned}
& \sum_{k=0}^{n}(-1)^{k} V_{r}(n, n-k) z^{k}=\prod_{\ell=0}^{n-1}\left(1+\left((2 \ell+1)^{2}+r\right) z\right) . \\
& \sum_{n \geq k} V_{r}(n, k) z^{n-k}=\prod_{\ell=0}^{k}\left(\left(1-(2 \ell+1)^{2}+r\right) z\right)^{-1}, \quad k \geq 0 .
\end{aligned}
$$

Proof. Replacing $x$ by $z^{-1}$ in (7), and multiplying both sides by $z^{n}$, gives

$$
\sum_{k=0}^{n} v_{r}(n, k) z^{n-k}=\prod_{\ell=0}^{n-1}\left(1-\left((2 \ell+1)^{2}+r\right) z\right)
$$

an hence replace $z$ by $-z$,

$$
\sum_{k=0}^{n}(-1)^{n-k} v_{r}(n, k) z^{n-k}=\prod_{\ell=0}^{n-1}\left(1+\left((2 \ell+1)^{2}+r\right) z\right)
$$

then replacing $k$ by $n-k$ gives (21). For (22), let $V_{r}^{(k)}(z)=\sum_{n \geq k} V_{r}(n, k) z^{n}$, hence the initial condition is given by

$$
V_{r}^{(0)}(z)=\sum_{n \geq k} V_{r}(n, 0) z^{n}=\sum_{n \geq k}(1+r)^{n} z^{n}=(1-(1+r) z)^{-1} .
$$

By virtue of (11),

$$
\sum_{n \geq k} V_{r}(n, k) z^{n}=\sum_{n \geq k} V_{r}(n-1, k-1) z^{n}+\left((2 k+1)^{2}+r\right) \sum_{n \geq k} V_{r}(n-1, k) z^{n}, \quad k \geq 1
$$

hence

$$
V_{r}^{(k)}(z)=z V_{r}^{(k-1)}(z)+\left((2 k+1)^{2}+r\right) z V_{r}^{(k)}(z), \quad k \geq 1,
$$




$$
V_{r}^{(k)}(z)=\frac{z}{1-\left((2 k+1)^{2}+r\right) z} V_{r}^{(k-1)}(z), \quad k \geq 1 .
$$

Iterating this recurrence, gives (22).

For a set of variables $y_{1}, y_{2}, \cdots, y_{n}$, the $k$-th elementary symmetric function $e_{k}\left(y_{1}, y_{2}, \cdots, y_{n}\right)$ and the $k$-th complete homogeneous symmetric function $h_{k}\left(y_{1}, y_{2}, \cdots, y_{n}\right)$ are given, respectively, by

$$
\begin{gathered}
e_{k}\left(y_{1}, y_{2}, \cdots, y_{n}\right)=\sum_{1 \leq \ell_{1}<\ell_{2}<\cdots<\ell_{k} \leq n} \prod_{i=1}^{k} y_{\ell_{i}}, \quad 1 \leq k \leq n \\
h_{k}\left(y_{1}, y_{2}, \cdots, y_{n}\right)=\sum_{1 \leq \ell_{1} \leq \ell_{2} \leq \cdots \leq \ell_{k} \leq n} \prod_{i=1}^{k} y_{\ell_{i}}, \quad k \geq 1
\end{gathered}
$$

with $e_{0}\left(y_{1}, y_{2}, \cdots, y_{n}\right)=h_{0}\left(y_{1}, y_{2}, \cdots, y_{n}\right)=1$, and $e_{k}\left(y_{1}, y_{2}, \cdots, y_{n}\right)=0$ for $k>n$ or $k<0$.

The generating functions of $e_{k}$ and $h_{k}$ are given by, see [7]

$$
\begin{aligned}
\sum_{k=0}^{n} e_{k}\left(y_{1}, y_{2}, \cdots, y_{n}\right) z^{k} & =\prod_{\ell=1}^{n}\left(1+y_{\ell} z\right) . \\
\sum_{k=0}^{n} h_{k}\left(y_{1}, y_{2}, \cdots, y_{n}\right) z^{k} & =\prod_{\ell=1}^{n}\left(1-y_{\ell} z\right)^{-1} .
\end{aligned}
$$

Using (21) and (22), it is not difficult to show that $v_{r}(n, k)$ and $V_{r}(n, k)$ are the specializations of the elementary and complete symmetric functions, i.e.,

$$
\begin{gathered}
v_{r}(n, n-k)=(-1)^{k} e_{k}\left(1^{2}+r, 3^{2}+r, \cdots,(2 n-1)^{2}+r\right), \\
V_{r}(n+k, n)=h_{k}\left(1^{2}+r, 3^{2}+r, \cdots,(2 n+1)^{2}+r\right) .
\end{gathered}
$$

In particular, at $r=0$, the central factorial numbers with odd arguments of the first kind are the elementary symmetric functions of the numbers $1^{2}, 3^{2}, \cdots,(2 n-1)^{2}$, i.e.,

$$
v(n, n-k)=(-1)^{k} e_{k}\left(1^{2}, 3^{2}, \cdots,(2 n-1)^{2}\right),
$$

and the central factorial numbers with odd arguments of the second kind are the complete homogeneous symmetric functions of the numbers $1^{2}, 3^{2}, \cdots,(2 n+1)^{2}$, i.e.,

$$
V(n+k, n)=h_{k}\left(1^{2}, 3^{2}, \cdots,(2 n+1)^{2}\right),
$$

Theorem 6. (Merca [8]) Let $k$ and $n$ be two positive integers, then

$$
e_{k}\left(y_{1}+t, y_{2}+t, \cdots, y_{n}+t\right)=\sum_{\ell=0}^{k}\left(\begin{array}{l}
n-\ell \\
k-\ell
\end{array}\right) e_{\ell}\left(y_{1}, y_{2}, \cdots, y_{n}\right) t^{k-\ell},
$$

and

$$
h_{k}\left(y_{1}+t, y_{2}+t, \cdots, y_{n}+t\right)=\sum_{\ell=0}^{k}\left(\begin{array}{c}
n-1+k \\
k-\ell
\end{array}\right) h_{\ell}\left(y_{1}, y_{2}, \cdots, y_{n}\right) t^{k-\ell},
$$

where $t, y_{1}, y_{2}, \cdots, y_{n}$ are variables. 
In the next theorem, we prove that the central factorial numbers with odd arguments can be expressed in terms of $r$-central factorial numbers with odd arguments and vice versa.

Theorem 7. For $n, k, r \geq 0$, we have

1) $v_{r}(n, k)=\sum_{\ell=k}^{n}\left(\begin{array}{l}\ell \\ k\end{array}\right)(-r)^{\ell-k} v(n, \ell)$,

2) $v(n, k)=\sum_{\ell=k}^{n}\left(\begin{array}{l}\ell \\ k\end{array}\right)(r)^{\ell-k} v_{r}(n, \ell)$,

3) $V_{r}(n, k)=\sum_{\ell=k}^{n}\left(\begin{array}{l}n \\ \ell\end{array}\right) V(\ell, k) r^{n-\ell}$

4) $V(n, k)=\sum_{\ell=k}^{n}\left(\begin{array}{l}n \\ \ell\end{array}\right)(-r)^{n-\ell} V_{r}(\ell, k)$.

Proof. By using (25) and Equation (29)

$$
\begin{aligned}
v_{r}(n, n-k) & =(-1)^{k} e_{k}\left(1^{2}+r, 3^{2}+r, \cdots,(2 n-1)^{2}+r\right) \\
& =(-1)^{k} \sum_{\ell=0}^{k}\left(\begin{array}{l}
n-\ell \\
k-\ell
\end{array}\right) e_{\ell}\left(1,3^{2}, \cdots,(2 n-1)^{2}\right) r^{k-\ell} \\
& =\sum_{\ell=0}^{k}\left(\begin{array}{l}
n-\ell \\
k-\ell
\end{array}\right)(-1)^{\ell} e_{\ell}\left(1,3^{2}, \cdots,(2 n-1)^{2}\right)(-r)^{k-\ell} \\
& =\sum_{\ell=0}^{k}\left(\begin{array}{l}
n-\ell \\
k-\ell
\end{array}\right) v(n, n-\ell)(-r)^{k-\ell} .
\end{aligned}
$$

Replacing $k$ by $n-k$,

$$
\begin{aligned}
v_{r}(n, k) & =\sum_{\ell=0}^{n-k}\left(\begin{array}{c}
n-\ell \\
n-k-\ell
\end{array}\right) v(n, n-\ell)(-r)^{n-k-\ell} \\
& =\sum_{\ell=k}^{n}\left(\begin{array}{c}
\ell \\
\ell-k
\end{array}\right) v(n, \ell)(-r)^{\ell-k},
\end{aligned}
$$

gives the first identity. From (27) and (29), we get

$$
\begin{aligned}
v(n, n-k) & =(-1)^{k} e_{k}\left(1^{2}, 3^{2}, \cdots,(2 n-1)^{2}\right) \\
& =(-1)^{k} \sum_{\ell=0}^{k}\left(\begin{array}{l}
n-\ell \\
k-\ell
\end{array}\right) e_{\ell}\left(1^{2}+r, 3^{2}+r, \cdots,(2 n-1)^{2}+r\right)(-r)^{k-\ell} \\
& =\sum_{\ell=0}^{k}\left(\begin{array}{l}
n-\ell \\
k-\ell
\end{array}\right)(r)^{k-\ell} v_{r}(n, n-\ell),
\end{aligned}
$$

By replacing $k$ by $n-k$ and then $n-\ell$ by $\ell$, we get the second identity. The last two identities can be proven similarly by using the relations (26), (28) and (30).

\section{The Generalized Central Factorial Matrices with Odd Arguments}

Matrix representation and factorization for the special numbers are well developed by many authors, see for example [5] [8] [9] [10] [11]. In the following, we define the $r$-central factorial matrices with odd arguments of both kinds and give factorizations for them. 
Definition 2. The r-central factorial matrix with odd arguments of the first kind is the $n \times n$ matrix defined by

$$
\mathcal{V}_{1}(n):=\mathcal{V}_{1}^{(r)}(n)=\left[\mathcal{V}_{r}(i, j)\right]_{0 \leq i, j \leq n-1},
$$

Similarly, the r-central factorial matrix with odd arguments of the second kind is the $n \times n$ matrix defined by

$$
\mathcal{V}_{2}(n):=\mathcal{V}_{2}^{(r)}(n)=\left[V_{r}(i, j)\right]_{0 \leq i, j \leq n-1}
$$

When $r=0$, we obtain the central factorial matrices with odd arguments of both kinds,

$$
\mathcal{M}_{1}(n)=[v(i, j)]_{0 \leq i, j \leq n-1}, \quad \text { and } \quad \mathcal{M}_{2}(n)=[V(i, j)]_{0 \leq i, j \leq n-1} .
$$

For example,

$$
\mathcal{V}_{1}(4)=\left[\begin{array}{cccc}
1 & 0 & 0 & 0 \\
-r-1 & 1 & 0 & 0 \\
r^{2}+10 r+9 & -2 r-10 & 1 & 0 \\
-r^{3}-35 r^{2}-259 r-225 & 3 r^{2}+70 r+259 & -3 r-35 & 1
\end{array}\right],
$$

and

$$
\mathcal{V}_{2}(4)=\left[\begin{array}{cccc}
1 & 0 & 0 & 0 \\
1+r & 1 & 0 & 0 \\
(1+r)^{2} & 2 r+10 & 1 & 0 \\
(1+r)^{3} & 3 r^{2}+30 r+91 & 3 r+35 & 1
\end{array}\right] .
$$

The orthogonality property (9) gives the following identity

$$
\left(\mathcal{V}_{1}(n)\right)^{-1}=\mathcal{V}_{2}(n), \quad n \geq 1
$$

The generalized $n \times n$ Pascal matrix $P_{n}[x]$ (see [12]) is defined as:

$$
P_{n}[x]=\left[\left(\begin{array}{c}
i \\
j
\end{array}\right) x^{i-j}\right]_{0 \leq i, j \leq n-1},
$$

with $P_{n}=P_{n}[1]$, the Pascal matrix of order $n$. Moreover,

$$
P_{n}^{-1}[x]=P_{n}[-x]=\left[(-1)^{i-j}\left(\begin{array}{l}
i \\
j
\end{array}\right) x^{i-j}\right]_{0 \leq i, j \leq n-1}
$$

From Theorem 7, we have the important matrix representations

$$
\mathcal{V}_{1}(n)=\mathcal{M}_{1}(n) P_{n}[-r], \quad n \geq 1,
$$

and

$$
\mathcal{V}_{2}(n)=P_{n}[r] \mathcal{M}_{2}(n), \quad n \geq 1
$$

For example

$$
V_{1}(4)=\left[\begin{array}{cccc}
1 & 0 & 0 & 0 \\
-1 & 1 & 0 & 0 \\
9 & -10 & 1 & 0 \\
225 & 259 & -35 & 1
\end{array}\right] \times\left[\begin{array}{ccccc}
1 & 0 & 0 & 0 & 0 \\
-r & 1 & 0 & 0 & 0 \\
r^{2} & -2 r & 1 & 0 & 0 \\
-r^{3} & 3 r^{2} & -3 r & 1 & 0
\end{array}\right]=\mathcal{M}_{1}(4) P_{4}[-r] .
$$

and 


$$
\mathcal{V}_{2}(4)=\left[\begin{array}{ccccc}
1 & 0 & 0 & 0 & 0 \\
r & 1 & 0 & 0 & 0 \\
r^{2} & 2 r & 1 & 0 & 0 \\
r^{3} & 3 r^{2} & 3 r & 1 & 0
\end{array}\right] \times\left[\begin{array}{cccc}
1 & 0 & 0 & 0 \\
1 & 1 & 0 & 0 \\
1 & 10 & 1 & 0 \\
1 & 91 & 35 & 10
\end{array}\right]=P_{5}[r] \mathcal{M}_{2}(5)
$$

\section{The Generalized Central Factorial Numbers and Legender-Stirling Numbers}

The Legendre-Stirling numbers were introduced by [13], and many properties of these numbers have been studied later in [14] [15].

The Legendre-Stirling numbers of the first kind $P s_{n}^{k}$ are defined by

$$
\prod_{j=0}^{n-1}(x-j(j+1))=\sum_{k=0}^{n} P s_{n}^{k} x^{k},
$$

and the Legendre-Stirling numbers of the second kind $P S_{n}^{k}$ are defined by

$$
x^{n}=\sum_{k=0}^{n} P S_{n}^{k} \prod_{j=0}^{k-1}(x-j(j+1)) .
$$

In fact, the Legendre-Stirling numbers are specializations of the elementary and complete homogeneous symmetric functions, i.e.,

$$
\begin{gathered}
P s_{n}^{n-k}=(-1)^{k} e_{k}(2,6, \cdots, n(n-1)), \\
P S_{n+k}^{n}=h_{k}(2,6, \cdots, n(n+1)) .
\end{gathered}
$$

We next give some connections between the $r$-central factorial numbers with odd arguments and the Legendre-Stirling numbers.

Theorem 8. For $n, k, r \geq 0$,

$$
\begin{gathered}
P s_{n}^{k}=\frac{1}{4^{n-k}} \sum_{i=k}^{n} \sum_{\ell=i}^{n}\left(\begin{array}{l}
i \\
k
\end{array}\right)\left(\begin{array}{l}
\ell \\
i
\end{array}\right) r^{\ell-i} v_{r}(n, \ell) \\
v_{r}(n, k)=\sum_{i=k}^{n} \sum_{\ell=i}^{n}\left(\begin{array}{l}
i \\
k
\end{array}\right)\left(\begin{array}{l}
\ell \\
i
\end{array}\right)(-1)^{\ell-k} 4^{n-\ell} r^{i-k} P s_{n}^{\ell} \\
P S_{n}^{k}=\frac{1}{4^{n-k}} \sum_{i=k}^{n} \sum_{\ell=k}^{i}(-1)^{n-\ell}\left(\begin{array}{l}
n \\
i
\end{array}\right)\left(\begin{array}{l}
i \\
\ell
\end{array}\right) r^{i-\ell} V_{r}(\ell, k) \\
V_{r}(n, k)=\sum_{i=k}^{n} \sum_{\ell=k}^{i}\left(\begin{array}{l}
n \\
i
\end{array}\right)\left(\begin{array}{l}
i \\
\ell
\end{array}\right) 4^{\ell-k} r^{n-i} P S_{\ell}^{k} .
\end{gathered}
$$

Proof. For (36), we note that

$$
\begin{aligned}
P s_{n}^{n-k} & =(-1)^{k} e_{k}(0,2, \cdots, n(n-1)) \\
& =(-1)^{k} \sum_{i=0}^{k}\left(\begin{array}{l}
n-i \\
k-i
\end{array}\right) e_{i}\left(\frac{1}{4}, 2+\frac{1}{4}, \cdots, n(n-1)+\frac{1}{4}\right)\left(\frac{-1}{4}\right)^{k-i} \\
& =(-1)^{k} \sum_{i=0}^{k}\left(\begin{array}{l}
n-i \\
k-i
\end{array}\right)\left(\frac{1}{4}\right)^{i} e_{i}\left(1,3^{2}, \cdots,(2 n-1)^{2}\right)\left(\frac{-1}{4}\right)^{k-i} \\
& =\frac{1}{4^{k}} \sum_{i=0}^{k} \sum_{\ell=0}^{i}\left(\begin{array}{l}
n-i \\
k-i
\end{array}\right)\left(\begin{array}{c}
n-\ell \\
i-\ell
\end{array}\right)(-1)^{\ell} e_{\ell}\left(1+r, 3^{2}+r, \cdots,(2 n-1)^{2}+r\right) r^{i-\ell} \\
& =\frac{1}{4^{k}} \sum_{i=0}^{k} \sum_{\ell=0}^{i}\left(\begin{array}{l}
n-i \\
k-i
\end{array}\right)\left(\begin{array}{c}
n-\ell \\
i-\ell
\end{array}\right) r^{i-\ell} v_{r}(n, n-\ell) .
\end{aligned}
$$


Then

$$
\begin{aligned}
P s_{n}^{k} & =\frac{1}{4^{n-k}} \sum_{i=0}^{n-k} \sum_{\ell=0}^{i}\left(\begin{array}{c}
n-i \\
n-k-i
\end{array}\right)\left(\begin{array}{c}
n-\ell \\
i-\ell
\end{array}\right) r^{i-\ell} v_{r}(n, n-\ell) \\
& =\frac{1}{4^{n-k}} \sum_{i=k}^{n} \sum_{\ell=0}^{n-i}\left(\begin{array}{c}
i \\
i-k
\end{array}\right)\left(\begin{array}{c}
n-\ell \\
n-i-\ell
\end{array}\right) r^{n-i-\ell} v_{r}(n, n-\ell) \\
& =\frac{1}{4^{n-k}} \sum_{i=k}^{n} \sum_{\ell=i}^{n}\left(\begin{array}{c}
i \\
k
\end{array}\right)\left(\begin{array}{c}
\ell \\
\ell-i
\end{array}\right) r^{\ell-i} v_{r}(n, \ell) .
\end{aligned}
$$

For (37), by virtue of (25),

$$
\begin{aligned}
v_{r}(n, n-k) & =(-1)^{k} e_{k}\left(1^{2}+r, 3^{2}+r, \cdots,(2 n-1)^{2}+r\right) \\
& =(-1)^{k} \sum_{i=0}^{k}\left(\begin{array}{l}
n-i \\
k-i
\end{array}\right) e_{i}\left(1,3^{2}, \cdots,(2 n-1)^{2}\right) r^{k-i} \\
& =(-1)^{k} \sum_{i=0}^{k}\left(\begin{array}{l}
n-i \\
k-i
\end{array}\right) 4^{i} e_{i}\left(\frac{1}{4}, \frac{9}{4}, \cdots, \frac{(2 n-1)^{2}}{4}\right) r^{k-i} \\
& =(-1)^{k} \sum_{i=0}^{k}\left(\begin{array}{l}
n-i \\
k-i
\end{array}\right) \sum_{\ell=0}^{i}\left(\begin{array}{c}
n-\ell \\
i-\ell
\end{array}\right) 4^{i} e_{\ell}(0,2, \cdots, n(n-1))\left(\frac{1}{4}\right)^{i-\ell} r^{k-i} \\
& =(-1)^{k} \sum_{i=0}^{k} \sum_{\ell=0}^{i}\left(\begin{array}{l}
n-i \\
k-i
\end{array}\right)\left(\begin{array}{c}
n-\ell \\
i-\ell
\end{array}\right)(-1)^{\ell} 4^{\ell} r^{k-i} P s_{n}^{n-\ell} .
\end{aligned}
$$

Hence

$$
\begin{aligned}
v_{r}(n, k) & =(-1)^{n-k} \sum_{i=0}^{n-k} \sum_{\ell=0}^{i}\left(\begin{array}{c}
n-i \\
n-k-i
\end{array}\right)\left(\begin{array}{c}
n-\ell \\
i-\ell
\end{array}\right)(-1)^{\ell} 4^{\ell} r^{n-k-i} P s_{n}^{n-\ell} \\
& =(-1)^{n-k} \sum_{i=k}^{n} \sum_{\ell=0}^{n-i}\left(\begin{array}{c}
i \\
i-k
\end{array}\right)\left(\begin{array}{c}
n-\ell \\
n-i-\ell
\end{array}\right)(-1)^{-\ell} 4^{\ell} r^{i-k} P s_{n}^{n-\ell} \\
& =\sum_{i=k}^{n} \sum_{\ell=i}^{n}\left(\begin{array}{l}
i \\
k
\end{array}\right)\left(\begin{array}{l}
\ell \\
i
\end{array}\right)(-1)^{\ell-k} 4^{n-\ell} r^{i-k} P s_{n}^{\ell} .
\end{aligned}
$$

The proofs of (38) and (39) are similar.

For example, for $n=3, k=2$, from (37) we have

$$
v_{r}(3,2)=\sum_{i=2}^{4} \sum_{l=i}^{3}\left(\begin{array}{l}
i \\
2
\end{array}\right)\left(\begin{array}{l}
l \\
i
\end{array}\right)(-1)^{l-2} 4^{3-l} r^{i-2} P s_{3}^{l}=-35-3 r,
$$

and for (36),

$$
P s_{3}^{2}=\frac{1}{4} \sum_{i=2}^{3} \sum_{l=i}^{3}\left(\begin{array}{l}
i \\
2
\end{array}\right)\left(\begin{array}{l}
l \\
i
\end{array}\right) r^{l-i} v_{r}(3, l)=-8,
$$

For example, for $n=4, k=3$, from (39) we have

$$
V_{r}(4,3)=\sum_{i=3}^{4} \sum_{l=3}^{i}\left(\begin{array}{l}
4 \\
i
\end{array}\right)\left(\begin{array}{l}
i \\
l
\end{array}\right) 4^{l-3} r^{4-i} P S_{l}^{3}=4 r+84,
$$

and for (38),

$$
P S_{4}^{3}=\frac{1}{4} \sum_{i=3}^{4} \sum_{l=3}^{i}(-1)^{4-l}\left(\begin{array}{l}
4 \\
i
\end{array}\right)\left(\begin{array}{l}
i \\
l
\end{array}\right) r^{i-l} V_{r}(l, 3)=20 .
$$




\section{Conclusion}

The $r$-central factorial numbers with odd arguments of both kinds are defined. We obtained recurrence relations, generating functions and explicit formulas of these numbers. Matrix representation and the relation between these numbers and Pascal matrix are given. The distribution of the signless $r$-central factorial numbers of odd arguments of the first kind is derived. Finally, connections between the $r$-central factorial numbers with odd arguments and the Legendre-Stirling numbers are investigated.

\section{Conflicts of Interest}

The authors declare no conflicts of interest regarding the publication of this paper.

\section{References}

[1] Riordan, J. (1968) Combinatorial Identities. John Wiley \& Sons, Inc., New York.

[2] Butzer, P.L., Schmidt, K., Stark, E.L. and Vogt, L. (1989) Central Factorial Numbers, Their Main Properties and Some Applications. Numerical Functional Analysis and Optimization, 10, 419-488. https://doi.org/10.1080/01630568908816313

[3] Kim, D.S., Dolgy, D.V., Kim, D. and Kim, T. (2019) Some Identities on R-Central Factorial Numbers and R-Central Bell Polynomials. Advances in Difference Equations, 2019, Article No. 245. https://doi.org/10.1186/s13662-019-1963-1

[4] Gelineau, Y. and Zeng, J. (2010) Combinatorial Interpretations of the Jacobi-Stirling Numbers. The Electronic Journal of Combinatorics, 17, R70. https://doi.org/10.37236/342

[5] Shiha, F.A. (2020) The r-Central Factorial Numbers with Even Indices. Advances in Difference Equations.

[6] Mansour, T., Mulay, S. and Shattuck, M. (2012) A General Two-Term Recurrence and Its Solution. The Electronic Journal of Combinatorics, 33, 20-26. https://doi.org/10.1016/j.ejc.2011.07.008

[7] Ramrez, J.L. and Shattuck, M. (2016) Generalized R-Whitney Numbers of the First Kind. Annales Mathematicae et Informaticae, 46, 175-193.

[8] Merca, M. (2013) A Note on the R-Whitney Numbers of Dowling Lattices. Comptes Rendus de I Académie des Sciences-Series I, 351, 649-655. https://doi.org/10.1016/j.crma.2013.09.011

[9] El-Desouky, B.S., Shiha, F.A. and Shokr, E.M. (2019) The Multiparameter R-Whitney Numbers. Filomat, 33, 931-943. https://doi.org/10.2298/FIL1903931E

[10] Mansour, T., Ramirez, J.L. and Shattuck, M. (2017) A Generalization of the R-Whitney Numbers of the Second Kind. Journal of Combinatorics, 8, 29-55. https://doi.org/10.4310/JOC.2017.v8.n1.a2

[11] Mezö, I. and Ramírez, J.L. (2015) The Linear Algebra of the R-Whitney Matrices. Integral Transforms and Special Functions, 26, 213-225. https://doi.org/10.1080/10652469.2014.984180

[12] Call, G.S. and Velleman, D.J. (1993) Pascal's Matrices. The American Mathematical Monthly, 100, 372-376. https://doi.org/10.1080/00029890.1993.11990415

[13] Everitt, W.N., Littlejohn, L.L. and Wellman, R. (2002) Legendre Polynomials, Legendre-Stirling Numbers, and the Left-Definite Spectral Analysis of the Legendre 
Differential Expression. Journal of Computational and Applied Mathematics, 148, 213-238. https://doi.org/10.1016/S0377-0427(02)00582-4

[14] Andrews, G.E. and Littlejohn, L.L. (2009) A Combinatorial Interpretation of the Legendre-Stirling Numbers. Proceedings AMS, 137, 2581-2590.

https://doi.org/10.1090/S0002-9939-09-09814-1

[15] Andrews, G.E., Gawronski, W. and Littlejohn, L.L. (2011) The Legendre-Stirling Numbers. Discrete Mathematics, 311, 1255-1272.

https://doi.org/10.1016/j.disc.2011.02.028 